\title{
Intermediate term outcomes after transvaginal uterine-preserving surgery in women with uterovaginal prolapse
}

\author{
Lisa C. Hickman ${ }^{1,2} \cdot$ Misha C. Tran $^{3} \cdot$ Marie Fidela R. Paraiso ${ }^{1}$. Mark D. Walters ${ }^{1} \cdot$ Cecile A. Ferrando $^{1}$
}

Received: 29 March 2021 / Accepted: 24 August 2021 / Published online: 29 September 2021

(C) The International Urogynecological Association 2021

\begin{abstract}
Introduction and hypothesis There is growing interest in and performance of uterine-preserving prolapse repairs. We hypothesized that there would be no difference in pelvic organ prolapse (POP) recurrence 2 years following transvaginal uterosacral ligament hysteropexy (USLH) and sacrospinous ligament hysteropexy (SSLH).

Methods This is a retrospective cohort study with a cross-sectional survey of women who underwent transvaginal uterinepreserving POP surgery from May 2016 to December 2017. Patients were included if they underwent either USLH or SSLH. POP recurrence was defined as a composite of subjective symptoms and/or retreatment. A cross-sectional survey was used to assess pelvic floor symptoms and patient satisfaction.

Results A total of 47 women met the criteria. Mean age was $52.8 \pm 12.5$ years, and all had a preoperative POP-Q stage of 2 $(55.3 \%)$ or $3(44.7 \%)$. Thirty $(63.8 \%)$ underwent SSLH and 17 (36.2\%) underwent USLH. There were no differences in patient characteristics or perioperative data. There was no difference in composite recurrence $(26.7 \%$ [8] vs $23.5 \%$ [4]) and retreatment $(6.7 \%$ [2] vs 0\%) retrospectively between SSLH and USLH groups at 22.6 months. Survey response rate was $80.9 \%$ (38) with a response time of 30.7 (28.0-36.6) months. The majority of patients (84.2\%) reported POP symptom improvement, and both groups reported great satisfaction (89.5\%). In respondents, $13.2 \%$ (5) reported subjective recurrence and $5.3 \%$ (2) underwent retreatment, with no differences between hysteropexy types. There were no differences in other pelvic floor symptoms.

Conclusions Although 1 in 4 women experienced subjective POP recurrence after transvaginal uterine-preserving prolapse repair and $<5 \%$ underwent retreatment at 2 years, our results must be interpreted with caution given our small sample size. No differences in outcomes were identified between hysteropexy types; however, additional studies should be performed to confirm these findings. Both hysteropexy approaches were associated with great patient satisfaction.
\end{abstract}

Keywords Hysteropexy $\cdot$ Native tissue repair $\cdot$ Pelvic organ prolapse $\cdot$ Sacrospinous ligament hysteropexy $\cdot$ Surgical outcomes $\cdot$ Uterosacral ligament hysteropexy

This research was presented at the American Urogynecologic Society's PFD Week 2020, Vancouver, Canada, 6-10 October 2020

Lisa C. Hickman

Lisa.Hickman@osumc.edu

1 Section of Urogynecology and Reconstructive Pelvic Surgery, Obstetrics, Gynecology \& Women's Health Institute, Cleveland Clinic Foundation, Cleveland, OH, USA

2 Division of Female Pelvic Medicine and Reconstructive Surgery, The Ohio State University Wexner Medical Center, 395 W. 12th Avenue, Room 504, OH 43210 Columbus, USA

3 University of Chicago School of Medicine, Chicago, IL, USA

\section{Introduction}

Pelvic organ prolapse (POP) is one of the most common hysterectomy indications for benign disease, accounting annually for nearly $20 \%$ of hysterectomies in the USA $[1,2]$. However, in recent years, the necessity of concomitant hysterectomy at the time of prolapse repair has come into question, with a growing body of evidence supporting the efficacy of uterine-conserving prolapse repair techniques. There is also increasing awareness among providers regarding patient attitudes towards hysterectomy and desire for uterine preservation [3, 4]. Together, this has translated into a greater interest in uterineconserving prolapse repairs and increasing rates of hysteropexies being performed in the USA [5]. 
Uterine-sparing procedures for prolapse repair can be approached either vaginally or abdominally. In 2017, the most commonly performed uterine-sparing procedures were uterosacral ligament hysteropexy (USLH), sacrospinous ligament hysteropexy (SSLH), and sacrohysteropexy [6]. The SSLH is performed using a vaginal approach; the sacrohysteropexy is approached abdominally; and the USLH can be performed vaginally or abdominally. A vaginal approach has several clear advantages over an abdominal approach: shorter operative and recovery times, improved esthetic outcomes, and less blood loss [7]. Of the two vaginal approaches, SSLH (with or without mesh augmentation) is the best-studied hysteropexy technique in the literature. The technique has consistently been shown to have similar anatomical outcomes and recurrence rates (based on pelvic organ prolapse quantification [POP-Q] staging) and similar improvements in quality of life and functioning compared with hysterectomybased native tissue repairs [8-11]. One difference between the two approaches is that a unilateral SSLH changes the axis of the vagina, deviating it unilaterally; however, it is unclear if there are any clinical implications associated with this anatomical change $[6,12]$. In contrast, USLH may result in a more anatomically correct repair of prolapse given the bilateral nature of the suspension. Furthermore, proponents of this surgical approach emphasize the uterosacral ligament's physiological role in uterine support and its high tensile strength [6].

Compared with the other hysteropexy techniques, only a small number of studies have assessed the efficacy and outcomes associated with USLH using a vaginal approach [13-16]. To date, there have been to our knowledge no studies comparing women who underwent transvaginal USLH to SSLH. Therefore, the primary aim of this study is to describe the incidence of POP recurrence 2 years following transvaginal native tissue hysteropexy surgery. We hypothesized that there would be no difference in POP recurrence between the two groups. Our secondary aims are to compare the incidence of recurrence between women undergoing USLH and SSLH and to describe perioperative adverse events, pelvic floor symptoms, and patient satisfaction following surgery.

\section{Materials and methods}

This is a retrospective cohort study of women with uterine prolapse who underwent either a transvaginal USLH or SSLH at our academic institution between 1 May 2016 and 31 December 2017. As Current Procedural Terminology (CPT) codes for transvaginal hysteropexy do not exist, the following strategy was employed for patient identification: CPT codes 57,283 (intraperitoneal colpopexy) and 57,282 (extraperitoneal colpopexy) were utilized to identify all patients undergoing a vaginal native tissue apical repair.
Operative reports were then reviewed to identify those patients who underwent either a transvaginal USLH or a transvaginal SSLH. The Cleveland Clinic Institutional Review Board approved this study.

Eligibility criteria included women aged $\geq 18$ at the time of initial POP surgery with documented stage $2-4$ uterine prolapse preoperatively. Patients were excluded if they had a history of previous POP surgery, had either a concurrent transvaginal mesh placement for prolapse or a concurrent hysterectomy, or if they were unable to comprehend English, as required for the cross-sectional component of the study.

The technique utilized for the SSLH was as follows: a semi-lunar incision was made inferior to the cervix. An extraperitoneal dissection was then performed using a combination of sharp and blunt dissection to access the right sacrospinous ligament. Two to three sutures were placed through the ligament with the assistance of a disposable suture-capturing device. Surgeons were permitted to utilize their preferred suture, which included either entirely polydioxanone or a combination of polydioxanone and prolene (generally 2:1). The sutures were then anchored through the posterior cervical stroma/uterosacral ligament and vaginal epithelium unilaterally, and tied to suspend the uterus and cervix to the ligament. Care was taken to ensure that no suture bridges were present. For the USLH, the following technique was utilized. A semi-lunar incision was made inferior to the cervix. The perineum was entered sharply, providing access to the abdominal cavity. Bowel was packed cephalad using a moistened laparotomy sponge. The uterosacral ligament was grasped at approximately the level of the ischial spine with a long Allis clamp. Two sutures of polydioxanone were placed through the ligament immediately beneath the Allis clamp. The same procedure was performed on the contralateral side. Sutures were then anchored through the uterosacral ligament and cervical stroma, bilaterally and tied down to suspend the uterus and cervix. For both hysteropexy techniques, additional procedures were at the discretion of the surgeon.

The system-wide electronic medical record (EMR) was queried for the retrospective portion of this study, to obtain patient characteristics, preoperative examination findings, and perioperative data, as well as to review follow-up visits to evaluate whether or not patients developed recurrent prolapse. This review was performed by two individuals ( $\mathrm{LCH}$ and $\mathrm{MCT}$ ), and several internal quality control measures were utilized to minimize ascertainment bias, including a well-defined data collection tool and randomized chart audits, to ensure the accuracy of data collection. Recurrent POP was defined subjectively by any mention of vaginal bulge symptoms recorded in the EMR regardless of physical examination findings, or objectively by retreatment with either reoperation or pessary use for prolapse. Time to recurrence was defined as the interval between the index prolapse surgery and the initial report of 
subjective or objective recurrence in months. Follow-up duration was defined by the last gynecology or urogynecology evaluation in the EMR. Although the majority of patients presented for post-operative evaluation at 6 weeks, which is our standard of care, provider practices vary at our institution after that time regarding subsequent follow-up; thus, any further clinical evaluation was identified and reviewed.

To evaluate recurrent $\mathrm{POP} /$ pelvic floor symptoms, retreatment, and patient satisfaction, a cross-sectional approach was utilized. A letter was mailed to all patients explaining the purpose of the study with an opportunity to opt out of participation. Patients were then contacted by phone and validated questionnaires were administered, after obtaining informed consent, to evaluate for any current POP symptoms, urinary incontinence, defecatory dysfunction and/ or sexual dysfunction using the Pelvic Floor Disability Index20 (PFDI-20), the Incontinence Severity Index (ISI), the Pelvic Organ Prolapse Incontinence Sexual Questionnaire IUGA-Revised (PISQ-IR), and the Patient Global Impression of Improvement (PGI-I) [17-20]. Patients were asked questions about any visits or retreatment for POP outside of our health system, why they elected to have a uterinepreserving prolapse repair, and any subsequent pregnancies, when applicable. Their satisfaction with their index hysteropexy surgery was also assessed (see Supplement 1).

Study data were collected and managed using the Research Electronic Data Capture (REDCap) tool hosted at Cleveland Clinic. REDCap is a secure, web-based application designed to support data capture for research studies [21]. Categorical data were presented as $n(\%)$ with $95 \%$ confidence intervals and continuous variables presented as mean \pm standard deviation and median (range). Outcome measures were compared using either an analysis of variance or a Student's $t$ test for parametric continuous outcomes, a Kruskal-Wallis or MannWhitney $U$ test for nonparametric outcomes, and a Chisquared test for all categorical outcomes. A $p$ value $\leq 0.05$ was considered significant. Data from women not known to have experienced a treatment failure (those lost to followupand/or those who are unable to be surveyed) were censored at the last known time of treatment or date of follow-up. JMP 12.2 (SAS Institute, Cary, NC, USA) was the statistical package used to perform all analyses.

\section{Results}

Forty-seven women met the eligibility criteria. Patient characteristics are presented in Table 1. The mean patient age and BMI were $52.8 \pm 12.5$ years and $26.8 \pm 4.5 \mathrm{~kg} / \mathrm{m}^{2}$ respectively. Median vaginal parity was 2 (range 1-8). The majority of patients were post-menopausal $(63.8 \%)$ and nonsmokers (97.9\%). All patients had a preoperative POP-Q stage of either $2(55.3 \%)$ or $3(44.7 \%)$. Median (interquartile range) preoperative POP-Q points were as follows: $\mathrm{Ba} 1$ (0 to 2$), \mathrm{C}-3$ ( -5 to $-1.5)$, TVL 9 ( 8 to 9$), \mathrm{Bp}-1$ ( -2 to 0$)$, and $\mathrm{D}-6$ ( -7 to -5 ). The majority experienced concurrent stress urinary incontinence (SUI, 68.1\%). There were no differences in baseline characteristics between the groups.
Table 1 Patient characteristics, $N=47$

\begin{tabular}{lllll}
\hline & All subjects $(N=47)$ & SSLH $(N=30)$ & $\begin{array}{l}\text { USLH } \\
(N=17)\end{array}$ & $p$ value \\
\hline Age at surgery (years) & $52.8 \pm 12.5$ & $54.1 \pm 2.3$ & $50.4 \pm 3.0$ & 0.17 \\
Body mass index $\left(\mathrm{kg} / \mathrm{m}^{2}\right)$ & $26.8 \pm 4.5$ & $27.4 \pm 4.4$ & $25.9 \pm 4.8$ & 0.15 \\
Vaginal parity & $2(1-8)$ & $2(1-5)$ & $2(1-8)$ & 0.13 \\
Menopause & $63.8 \%(30)$ & $70.0 \%(21)$ & $52.9 \%(9)$ & 0.34 \\
Tobacco use & $2.1 \%(1)$ & $3.3 \%(1)$ & $0 \%(0)$ & 1.00 \\
Diabetes & $8.5 \%(4)$ & $10.0 \%(3)$ & $5.9 \%(1)$ & 1.00 \\
Coronary artery disease & $4.3 \%(2)$ & $3.3 \%(1)$ & $5.9 \%(1)$ & 1.00 \\
Asthma, COPD & $6.4 \%(3)$ & $6.7 \%(2)$ & $5.9 \%(1)$ & 1.00 \\
OSA/CPAP & $4.3 \%(2)$ & $6.7 \%(2)$ & $0 \%(0)$ & 0.53 \\
Chronic constipation & $38.3 \%(18)$ & $40.0 \%(12)$ & $35.3 \%(6)$ & 1.00 \\
Preoperative SUI & $68.1 \%(32)$ & $60.0 \%(18)$ & $82.4 \%(14)$ & 0.19 \\
Preoperative UUI & $34.0 \%(16)$ & $40.0 \%(12)$ & $23.5 \%(4)$ & 0.34 \\
POP stage & & & & \\
Stage 2 & $55.3 \%(26)$ & $63.3 \%(19)$ & $41.2 \%(7)$ & 0.22 \\
Stage 3 & $44.7 \%(21)$ & $36.7 \%(11)$ & $58.8 \%(10)$ & 0.22 \\
\hline
\end{tabular}

Data presented as mean $\pm \mathrm{SD}$, median (range), and \% (n)

SSLH sacrospinous ligament hysteropexy, USLH uterosacral ligament hysteropexy, COPD chronic obstructive pulmonary disease, $O S A / C P A P$ obstructive sleep apnea, continuous positive airway pressure, $S U I$ stress urinary incontinence, UUI urgency urinary incontinence 
Perioperative data are presented in Table 2. Of the patients, $63.8 \%$ underwent SSLH and $36.2 \%$ underwent USLH. The majority of women in both groups underwent concurrent anterior colporrhaphy (89.4\%), posterior colporrhaphy $(70.2 \%)$, and midurethral sling procedure $(51.1 \%)$. There were no differences in median case time (97 [range 85-114] min), estimated blood loss $(80 \pm 43 \mathrm{ml})$, intraoperative complications (2.1\% [1]), or post-operative complications (17.0\% [8]).

Retrospective recurrence data are outlined in Table 3. Median duration of follow-up with a gynecologist was 22.6 months. The overall composite POP recurrence incidence was $25.5 \%(12,95 \%$ CI $16.7,33.1)$, with $4.3 \%$ (2, $95 \%$ CI 2.6, 6.9) undergoing retreatment. There was no difference in overall composite POP recurrence and retreatment incidence between the SSLH and USLH groups $(26.7 \%$ (8) vs $23.5 \%(4), p=1.00$ and $6.7 \%$ (2) vs $0 \%, p=.53$ respectively). Median time to recurrence was 316 (119-572) days with no significant differences between the groups. Only one patient in the SSLH group underwent subsequent hysterectomy as part of her reoperation for recurrent prolapse.

Thirty-eight women responded to the cross-sectional survey for a response rate of $80.9 \%$. There were no differences in the number of respondents from each hysteropexy group. The median time to survey response was 30.7 (28.0-36.6) months, with a longer time to survey result for women from the SSLH group compared than for those from the USLH group (33.3 [29.4-41.4] vs 22.8 [18.5-31.2], $p<0.001)$. There were no differences in patient characteristics between the women who responded to the survey and those who did not (data not shown).

When asked about reasons for choosing a hysteropexy over hysterectomy, most women, 15 , reported a desire to keep the uterus if there was no medical need for its removal. Other answers selected by patients included reduction in surgical risks (6), followed by desire to maintain fertility (3), importance for sexuality/sexual function (3), and a variety of "other" patient-specific reasons (7). The majority of women reported that their post-surgical condition was "better" (84.2\%) and endorsed satisfaction with their surgery $(89.5 \%)$ by affirmatively answering the question of whether they would undergo their surgery again. There was no difference in these responses between the hysteropexy groups.

From the cross-sectional survey, the overall prolapse recurrence incidence was found to be $13.2 \%(5,95 \%$ CI 9.4, 17.2), with $8.0 \%$ of women in the SSLH and $23.1 \%$ of women in the USLH groups meeting the composite definition (subjective and/or retreatment, $p=0.32$; Table 4). The overall retreatment incidence was $5.3 \%$, with $8.0 \%$ of women in the SSLH group and no women in the USLH group undergoing retreatment.

Inquiry regarding pelvic floor symptoms revealed that $36.8 \%$ (14) of women reported urgency urinary incontinence, $23.7 \%$ (9) reported stress urinary incontinence, and $15.8 \%$ (6) reported abdominopelvic pain. There was no difference in these findings between the hysteropexy groups. Of the women, $81.6 \%$ (31) reported current sexual activity, with $13.3 \%$ (4) experiencing dyspareunia, with no differences by hysteropexy approach. Last, 2 patients in the SSLH group each went on to have one subsequent pregnancy. Both delivered at term, 1 had a cesarean section and the other a spontaneous vaginal delivery.

\section{Discussion}

To our knowledge, this is the first study comparing intermediate-term outcomes between USLH and SSLH using a transvaginal approach. In this study, we found that although 1 in 4 women experienced subjective prolapse at 2 years
Table 2 Perioperative data, $N=$ 47

\begin{tabular}{lllll}
\hline & $\begin{array}{l}\text { All subjects } \\
(N=47)\end{array}$ & $\begin{array}{l}\text { SSLH } \\
(N=30)\end{array}$ & USLH $(N=17)$ & $p$ value \\
\hline Concomitant procedures & & & & \\
Anterior repair & $89.4 \%(42)$ & $86.7 \%(26)$ & $94.1 \%(16)$ & 0.64 \\
Posterior repair & $70.2 \%(33)$ & $63.3 \%(19)$ & $82.3 \%(14)$ & 0.20 \\
MUS procedure & $51.1 \%(24)$ & $46.7 \%(14)$ & $58.8 \%(10)$ & 0.55 \\
Intraoperative complications & $2.1 \%(1)$ & $3.3 \%(1)$ & $0 \%(0)$ & 1.00 \\
Case time (min) & $97(85-114)$ & $95(83-115)$ & $102(87-116)$ & 0.52 \\
EBL (cc) & $79.7 \pm 42.7$ & $76.7 \pm 78.7$ & $85.0 \pm 29.0$ & 0.77 \\
Length of stay (days) & $1(1-2)$ & $1(1-1)$ & $1(1-2)$ & 0.13 \\
Post-operative complications (with UTI) & $34.0 \%(16)$ & $33.3 \%(10)$ & $35.3(6)$ & 1.00 \\
Post-operative complications (without UTI) & $17.0 \%(8)$ & $20.0 \%(6)$ & $11.8 \%(2)$ & 0.69 \\
\hline
\end{tabular}

Data presented as mean $\pm \mathrm{SD}$, median (range) and \% $(n)$

SSLH sacrospinous ligament hysteropexy, USLH uterosacral ligament hysteropexy, MUS midurethral sling, $E B L$ estimated blood loss 
Table 3 Retrospective recurrence data, $N=47$

\begin{tabular}{|c|c|c|c|c|}
\hline & $\begin{array}{l}\text { All subjects } \\
(N=47)\end{array}$ & SSLH $(N=30)$ & $\operatorname{USLH}(N=17)$ & $p$ value \\
\hline Median follow-up with a gynecologist (months) & $22.6(4.1-28.9)$ & $22.4(5.2-29.2)$ & $23.3(1.5-29.8)$ & 0.41 \\
\hline \multicolumn{5}{|l|}{ Recurrence } \\
\hline Subjective & $25.5 \%(12)$ & $26.7 \%(8)$ & $23.5 \%(4)$ & 1.00 \\
\hline Retreatment & $4.3 \%(2)$ & $6.7 \%(2)$ & $0 \%(0)$ & 0.53 \\
\hline Composite & $25.5 \%(12)$ & $26.7 \%(8)$ & $23.5 \%(4)$ & 1.00 \\
\hline Time to recurrence & $316(119-572)$ & $340(129-574)$ & $62(48-845)$ & 0.45 \\
\hline De novo SUI & $4.3 \%(2)$ & $6.7 \%(2)$ & $0 \%(0)$ & 0.53 \\
\hline Recurrent or persistent SUI & $12.8 \%(6)$ & $16.7 \%(5)$ & $5.9 \%(1)$ & 0.40 \\
\hline De novo UUI & $10.6 \%(5)$ & $13.3 \%(4)$ & $5.9 \%(1)$ & 0.64 \\
\hline
\end{tabular}

Data presented as mean $\pm \mathrm{SD}$, median $(\mathrm{IQR})$ and $\%(n)$

SSLH sacrospinous ligament hysteropexy, USLH uterosacral ligament hysteropexy, SUI stress urinary incontinence, UUI urgency urinary incontinence

postoperatively, less than $5 \%$ underwent retreatment for their prolapse, and there was no difference in these findings by hysteropexy type in our small cohort of patients. Upon cross-sectional follow-up with patients at a median of 2.5 years after the initial hysteropexy, $13 \%$ of women met the subjective recurrence criteria and only 5\% had undergone retreatment, again with no significant differences between the surgical approaches. The majority of women reported satisfaction with the surgical procedure, regardless of hysteropexy approach, and nearly $85 \%$ stated that their preoperative condition was improved. Furthermore, no significant differences were found between the types of hysteropexy with regard to postoperative pelvic floor symptoms reported by survey respondents. Caution must be taken when interpreting our findings given the small sample size in this study, and our findings should be utilized for hypothesis generation and to aid in the design of a larger prospective cohort comparing these two groups.

Historically, patients with uterovaginal prolapse were counseled to undergo hysterectomy at the time of their prolapse repair. In recent years, the importance of removing the uterus in an effort to restore pelvic organ support has been questioned, as there has been a growing body of literature showing favorable outcomes following uterine-preserving prolapse repairs. A systematic review and meta-analysis by Meriwether et al. evaluated nearly 100 hysteropexy studies [22]. Although many of the studies included in the analysis reported on mesh-augmented prolapse repairs, a comparison of the vaginal native tissue prolapse repairs revealed shortened operative times, lower estimated blood loss, and no

Table 4 Cross-sectional survey data, $N=38$

\begin{tabular}{|c|c|c|c|c|}
\hline & $\begin{array}{l}\text { All respondents } \\
(N=38)\end{array}$ & $\begin{array}{l}\text { SSLH } \\
(N=25)\end{array}$ & $\begin{array}{l}\text { USLH } \\
(N=13)\end{array}$ & $p$ value \\
\hline Median time to survey result (months) & $30.7(28.0-36.6)$ & $33.3(29.4-41.4)$ & $22.8(18.5-31.2)$ & $<0.001$ \\
\hline Post-surgical condition (better) & $84.2 \%(32)$ & $76.0 \%(19)$ & $100 \%(13)$ & 0.08 \\
\hline Would have surgery again $(\%, n)$ & $89.5 \%(34)$ & $88.0 \%(22)$ & $92.3 \%(12)$ & 1.00 \\
\hline Recurrent vaginal bulge symptoms & $13.2 \%(5)$ & $8.0 \%(2)$ & $23.1 \%(3)$ & 0.32 \\
\hline Recurrence (retreatment: pessary or surgery) & $5.3 \%(2)$ & $8.0 \%(2)$ & $0 \%(0)$ & 0.54 \\
\hline Evaluation for SUI ${ }^{\mathrm{a}}$ & $10.5 \%(4)$ & $16.0 \%(4)$ & $0 \%(0)$ & 0.28 \\
\hline Current UUI & $36.8 \%(14)$ & $44.0 \%(11)$ & $23.1 \%(3)$ & 0.29 \\
\hline Current SUI & $23.7 \%(9)$ & $28.0 \%(7)$ & $15.4 \%(2)$ & 0.46 \\
\hline Abdominopelvic pain & $15.8 \%(6)$ & $16.0 \%(4)$ & $15.4 \%(2)$ & 1.00 \\
\hline Sexual activity (yes vs no) & $81.6 \%(31)$ & $80.0 \%(20)$ & $84.6 \%(11)$ & 1.00 \\
\hline Pain with intercourse (sometimes, usually, always) & $13.3 \%(4)$ & $15.8 \%(3)$ & $9.1 \%(1)$ & 1.00 \\
\hline
\end{tabular}

Data presented as mean $\pm \mathrm{SD}$, median (IQR), and \% ( $n$ )

${ }^{a}$ Persistent, recurrent, or de novo SUI 
differences in recurrence outcomes in patients undergoing hysteropexy procedures. In a second systematic review and meta-analysis by Meriwether et al., analysis of vaginal native tissue hysteropexy (SSLH, USLH, or "various") outcomes showed a subjective prolapse recurrence incidence of $3.3 \%$ and reoperation rate of $4.1 \%$ [23]. This incidence increased to $19.0 \%$ when "any definition" was used to study prolapse recurrence.

In our study, we found a similar incidence of retreatment reported in the aforementioned analysis, whereas our subjective recurrence incidence was significantly higher. Although this may not be related to the underlying reason for this finding, it is important to point out that in comparison with the review published by Meriwether et al. [22], we evaluated two different types of hysteropexy, whereas their data were largely driven by SSLH studies. Our higher subjective recurrence rate could be related to the broad definitions we used in our study for subjective recurrence. For the retrospective portion of this study, patients were considered to have a subjective recurrence if there was any recorded mention of vaginal bulge symptoms in the EMR, regardless of physical examination findings. Additionally, we used the Pelvic Organ Prolapse Distress Inventory 6 (POPDI-6) question "Do you usually have a bulge or something falling out that you can see or feel in your vaginal area?" in the prospective survey with the associated scale of bother. We elected to use a strict definition here as well, such that any positive response to this question, regardless of level of bother, was considered a subjective recurrence. We did, however, find that our composite recurrence incidence of $25 \%$, which was largely driven by the incidence of subjective recurrence, was similar to the "any definition" recurrence data, which permitted any stated recurrence definition reported by individual study authors (as opposed to repeat surgery for prolapse or prolapse recurrence symptoms), reported by Meriwether et al. [23]. These consistent findings provide us with data that we can use to counsel our patients properly, and also highlight the importance of patient-centered subjective outcomes when it comes to evaluating the efficacy of our prolapse surgeries.

As of late, patients are increasingly choosing or desiring uterine-preserving surgeries to treat their symptomatic prolapse [5]. When offered a surgical choice with similar outcomes, studies suggest that 36-60\% of women will choose a hysteropexy over hysterectomy [3, 24]. In our study, patients were asked about their reason for choosing hysteropexy. Similar to previously published studies, the majority of patients felt that the uterus should not be removed if there was not an important reason for doing so. We were also able to demonstrate that pregnancy is possible following hysteropexy surgery. Two patients in our study, both of whom had undergone SSLH, went on to carry term pregnancies with uncomplicated deliveries. Our findings are similar to that described by Wieslander et al. and add to the relative paucity of literature on pregnancy safety after prolapse repair [25].

This study also provides an important contribution to the limited data on USLH. A study by Romanzi and Tyagi retrospectively compared the outcomes of 200 women who had undergone either a transvaginal USLH or a vaginal hysterectomy with uterosacral ligament suspension at a median follow-up 1.5 years [14]. The authors found similar anatomical outcomes between the two surgical techniques, but reported no data on subjective recurrence or retreatment. In another retrospective study, Milani et al. followed 20 women who had undergone transvaginal USLH for an average of 33.2 months [15]. Anatomical recurrence, defined as $\geq$ stage 2 prolapse, was observed in $25 \%$ of patients, with $15 \%$ requiring reoperation. This same group retrospectively compared transvaginal USLH with hysterectomy and uterosacral ligament colpopexy in 104 patients [13]. At a mean 35-month follow-up, similar subjective and objective recurrence rates were found in the groups; however, there was a significantly higher reoperation rate in the hysteropexy group $(13.5 \%$ vs $1.9 \%, p=0.04)$. Although their reoperation rate was higher than what we reported in our study, their reported subjective recurrence incidence was in line with our findings. Last, Aserlind et al. retrospectively reviewed 40 patients who had undergone transvaginal USLH with an average follow-up time of 17.2 months and found significant anatomical improvements with no reoperation for recurrent POP [16]. At our institution, the USLH technique came into more common practice in 2016, utilizing a similar technique to uterosacral ligament colpopexy and as presented previously [26]. The median follow-up time after USLH in the present study was 23 months for the retrospective portion of the study, at which time no patients had undergone retreatment, and is similar to the study by Aserlind et al. [16].

Another important finding of this study was the lack of difference in the incidence of recurrence between SSLH and USLH. To our knowledge, no studies in the literature have compared these groups directly. There were no differences in patient characteristics or examination findings preoperatively between the groups, suggesting that either approach can be offered to patients with symptomatic prolapse, as long as total vaginal length will not preclude the uterus and cervix from reaching the SSL. The selection of the specific hysteropexy approach should be informed by patient-specific factors and surgeon expertise and must involve comprehensive counseling of the patient prior to surgery.

Strengths of this study include the well-characterized group of women who received care at our academic medical center by board-certified Female Pelvic Medicine and Reconstructive Surgery specialists. All retrospective data, which included a wide array of patient characteristics and perioperative outcomes, was obtained from our reliable EMR. Ascertainment bias was minimized through the use of 
a well-defined data collection tool and several internal quality control measures. Additionally, the survey response rate was high, at $81 \%$, with intermediate-term follow-up. This direct patient inquiry also helped to reduce reviewer/ documentation bias with our study outcomes. Last, validated surveys were utilized to evaluate for the presence of pelvic floor symptoms.

The most significant limitation of the study is the small sample sizes for the hysteropexy groups. This contributes to a lack of power and risk of a type 2 error. As such, we were unable to adequately test hypotheses and our findings should be interpreted with caution and utilized for hypothesis generation. Additional limitations of this research include the retrospective design and the biases associated with these studies. The lack of objective recurrence data is also a limitation, as having patients present for a repeat office examination was prohibited by the COVID-19 pandemic and a variety of other issues. Using our strict definition of subjective recurrence, a patient who reported the presence of bulge symptoms, yet had no significant prolapse on examination, was still considered to have a recurrence. It is possible with this definition that our composite POP recurrence incidence is artificially greater than that truly experienced by patients. A prospective study with subjective, objective and retreatment data will be important for better defining this population of women. Another limitation is the external validity of our data. All surgeries were performed at a single, large-volume institution on a relatively homogenous patient population, which may preclude more broad application of our findings. Finally, although this study provides valuable data on intermediate-term hysteropexy outcomes that can be used for perioperative patient counseling, longer-term studies with adequate power will be needed to better characterize the durability of transvaginal uterinepreserving prolapse repairs.

\section{Conclusions}

Two years following transvaginal native tissue hysteropexy surgery, 1 in 4 women experienced subjective POP recurrence; however, less than $5 \%$ underwent retreatment in our small retrospective cohort. It is important to interpret these findings with caution, however, given the sample size limitations in this study. Additionally, we found that there was similar efficacy between the SSLH and USLH cohorts, which should be further evaluated in future well-designed, prospective studies. When women were further assessed in a crosssectional manner, the incidence of retreatment remained 5\%, which supports our retrospective findings, but may also be subject to the limitations of our sample size, and these data should be utilized more for hypothesis generation. Importantly, both surgical approaches were associated with great patient satisfaction and improvement in pelvic floor symptoms.

Supplementary Information The online version contains supplementary material available at https://doi.org/10.1007/s00192-021-04987-5

\section{Declarations}

Financial disclaimer/conflicts of interest None.

\section{References}

1. Whiteman MK, Hillis SD, Jamieson DJ, et al. Inpatient hysterectomy surveillance in the United States, 2000-2004. Am J Obstet Gynecol. 2008;198(1):34.e1-7.

2. Wright JD, Herzog TJ, Tsui J, et al. Nationwide trends in the performance of inpatient hysterectomy in the United States. Obstet Gynecol. 2013;122(2):233-41.

3. Frick AC, Barber MD, Paraiso MFR, Ridgeway B, Jelovsek JE, Walters MD. Attitudes toward hysterectomy in women undergoing evaluation for Uterovaginal prolapse. Female Pelvic Med Reconstr Surg. 2013;19(2):103-9.

4. Good MM, Korbly N, Kassis NC, et al. Prolapse-related knowledge and attitudes toward the uterus in women with pelvic organ prolapse symptoms. Am J Obstet Gynecol. 2013;209(5):481.e1-6.

5. Madsen AM, Raker C, Sung VW. Trends in hysteropexy and apical support for uterovaginal prolapse in the United States from 2002 to 2012. Female Pelvic Med Reconstr Surg. 2017;23(6):365-71.

6. Ridgeway BM, Cadish L. Hysteropexy. Clin Obstet Gynecol. 2017;60(2):312-23.

7. Gutman R, Maher C. Uterine-preserving POP surgery. Int Urogynecol J. 2013;24(11):1803-13.

8. Maher CF, Cary MP, Slack MC, et al. Uterine preservation or hysterectomy at sacrospinous colpopexy for uterovaginal prolapse? Int Urogynecol J. 2001;12(6):381-4; discussion 384-5.

9. Dietz V, Huisman M, de Jong JM, et al. Functional outcome after sacrospinous hysteropexy for uterine descensus. Int Urogynecol J Pelvic Floor Dysfunct. 2008;19(6):747-52.

10. Dietz V, Van Der Vaart CH, Van Der Graaf Y, et al. One-year follow-up after sacrospinous hysteropexy and vaginal hysterectomy for uterine descent: a randomized study. Int Urogynecol J. 2010;21(2):209-16.

11. van Brummen HJ, van de Pol G, Aalders CIM, et al. Sacrospinous hysteropexy compared to vaginal hysterectomy as primary surgical treatment for a descensus uteri: effects on urinary symptoms. Int Urogynecol J Pelvic Floor Dysfunct. 2003;14(5):350-5; discussion 355.

12. Jeng C-J, Yang Y-C, Tzeng C-R, et al. Sexual functioning after vaginal hysterectomy or transvaginal sacrospinous uterine suspension for uterine prolapse: a comparison. J Reprod Med. 2005;50(9):669-74.

13. Milani R, Manodoro S, Cola A, et al. Transvaginal uterosacral ligament hysteropexy versus hysterectomy plus uterosacral ligament suspension: a matched cohort study. Int Urogynecol J. 2019;31(9):1867-72.

14. Romanzi LJ, Tyagi R. Hysteropexy compared to hysterectomy for uterine prolapse surgery: does durability differ? Int Urogynecol J. 2012;23(5):625-31. 
15. Milani R, Frigerio M, Manodoro S, et al. Transvaginal uterosacral ligament hysteropexy: a retrospective feasibility study. Int Urogynecol J. 2017;28(1):73-6.

16. Aserlind A, Garcia AN, Medina CA. Uterus-sparingsurgeryoutcomes of transvaginal uterosacral ligament hysteropexy. J Minim Invasive Gynecol. 2020;28(1):100-6.

17. Barber MD, Walters MD, Bump RC. Short forms of two conditionspecificquality-of-life questionnaires for women with pelvic floor disorders (PFDI-20 and PFIQ-7). Am J Obstet Gynecol. 2005;193(1):103-13.

18. Rogers RG, Coates KW, Kammerer-Doak D, et al. A short form of the pelvic organ prolapse/urinary incontinence sexual questionnaire (PISQ-12). Int Urogynecol J Pelvic Floor Dysfunct. 2003;14(3): 164-8; discussion 168.

19. Srikrishna S, Robinson D, Cardozo L. Validation of the patient global impression of improvement (PGI-I) for urogenital prolapse. Int Urogynecol J. 2010;21(5):523-8.

20. Sandvik H, Espuna M, Hunskaar S. Validity of the incontinence severity index: comparison with pad-weighing tests. Int Urogynecol J Pelvic Floor Dysfunct. 2006;17(5):520-4.

21. Harris PA, Taylor R, Thielke R, et al. Research electronic data capture (REDCap) - a metadata-driven methodology and workflow process for providing translational research informatics support. J Biomed Inform. 2009;42(2):377-81.
22. Meriwether KV, Antosh DD, Olivera CK, et al. Uterine preservation vs hysterectomy in pelvic organ prolapse surgery: a systematic review with meta-analysis and clinical practice guidelines. Am J Obstet Gynecol. 2018;219(2):129-146.e2.

23. Meriwether KV, Balk EM, Antosh DD, et al. Uterine-preserving surgeries for the repair of pelvic organ prolapse: a systematic review with meta-analysis and clinical practice guidelines. Int Urogynecol J. 2019;30(4):505-22.

24. Korbly NB, Kassis NC, Good MM, et al. Patient preferences for uterine preservation and hysterectomy in women with pelvic organ prolapse. Am J Obstet Gynecol. 2013;209(5):470.e1-6.

25. Wieslander CK, Roshanravan SM, Wai CY, Schaffer JI, Corton MM. Uterosacral ligament suspension sutures: anatomic relationships in unembalmed female cadavers. Am J Obstet Gynecol. 2007;197(6):672.e1-6.

26. Hickman LC, Tran MC, Thomas TN, et al. Vaginal uterosacral ligament hysteropexy: technique and clinical outcomes. In: AUGS PFD Week. Chicago, Illinois; Female Pelvic Med Reconstr Surg. 2018; 24(5S):S158.

Publisher's note Springer Nature remains neutral with regard to jurisdictional claims in published maps and institutional affiliations. 